\title{
Diagnostics of Machinery Faults based on EMD and ICA
}

\author{
Fengli Wang ${ }^{1, a}$, Yannian Cai ${ }^{2, b}$, Sihong $\mathrm{Li}^{3, \mathrm{c}}$, Hui Xing ${ }^{4, \mathrm{~d}}$ \\ ${ }^{1}$ College of Marine Engineering, Dalian Maritime University, Dalian, 116026, P. R. China \\ ${ }^{2}$ College of Marine Engineering, Dalian Maritime University, Dalian,116026, P. R. China \\ ${ }^{3}$ Technology Development Center, Guangdong Industry Technical College, \\ Guangzhou, 510300, P. R. China \\ ${ }^{4}$ College of Marine Engineering, Dalian Maritime University, Dalian, 116026, P. R. China \\ aemail: wangflsky997@sina.com, bemail:908414835@qq.com, ${ }^{\mathrm{c} e m a i l:}$ \\ sihongli@sina.com, demail:xingcage@sina.com
}

\begin{abstract}
Keywords: Empirical mode decomposition; Independent component analysis; Fault diagnosis; Rotating machinery.
\end{abstract}

\begin{abstract}
Diagnostics of the rotating machinery can identify potential failure at its early stage and reduce severe machine damage and costly machine downtime. Rub-impact is common faults in rotating machinery and results in impact and friction between rotor and stator. The vibration signals due to impact and friction are always non-stationary which includes the rub-impact signal, the background signal and the noise signal. Empirical mode decomposition (EMD) is based on the local characteristic time scale of signal and could decompose the complicated signal into a number of intrinsic mode functions (IMFs). Due to the weak rub-impact signal submerged in the background and noise signals, EMD procedure would generate the components redundancy. In order to solve the problem, a novel method combining with independent component analysis (ICA) and EMD is developed. ICA was introduced into EMD, so that the IMFs are orthogonal to each other and the components redundancy can be removed. Experimental analysis results show that the proposed method is more suitable and have a better performance for the incipient fault detection. The proposed method is thus proved to have potential to become a powerful tool for the surveillance and diagnosis of rotating machinery.
\end{abstract}

\section{Introduction}

The goal of health diagnosis is to identify potential failure caused at the early stage such that timely adjustment and maintenance actions can be taken to reduce severe machine damage and costly machine downtime [1-3]. Vibration signal analysis has long been an established method for condition monitoring of rotating machinery which covers a broad range of mechanical equipment and plays a significant role in industrial applications. It generally operates under tough working environment and is therefore subject to faults, which could be detected and diagnosed by using signal processing techniques. Rub fault is common faults in rotating machinery which can result in broken machine parts, and lead catastrophic breakdown of the rotating machinery. In order to avoid the occurrence of rub-impact, vibration signal analysis is widely used in rotating machinery condition monitoring and fault diagnosis. Usually, depending on machine operating conditions and severity of defects, the measured vibration signals are always non-stationary and modulated. Furthermore, when the local rub-impact occurs in the rotor system, the vibration signal includes the rub-impact signal, the background signal and the noise signal. The key of the rub-impact fault diagnosis is to extract the rub-impact feature from the vibration signal of the rotor system.

Empirical mode decomposition (EMD) as an adaptive method, is a powerful tool for analyzing any composite, nonlinear and non-stationary signals. EMD is based on the local characteristic time scale of signal and can decompose the complicated signal into a number of IMFs (intrinsic mode functions), which are band limited and can represent the features of signal and reserve the local information [4-7]. However, the decomposition algorithm has some implicit difficulties and the procedures create 
several drawbacks that originate strange decompositions. EMD cannot guarantee completeness and orthogonality, and may produce redundant components and affect the accuracy of the decompositions.

Independent component analysis (ICA) is a method for finding a linear representation of non-Gaussian so that the components are statistically independent, which is effective in removing components redundancy[8-10]. In this paper, the ICA was introduced into EMD to overcome the above limitations, thus the IMFs are orthogonal to each other and components redundancy can be removed effectively. Finally, the non-stationary signal can be decomposed accurately, and a much better decomposition performances can be obtained.

\section{EMD and Hilbert Transform}

EMD as an adaptive method, is developed from the simple assumption that any signal consists of different simple intrinsic modes of oscillations. With the EMD technique, any complicated signal can be decomposed into a collection of intrinsic mode functions (IMFs),, each of which must satisfy the following definition [4]: (1) In the entire data set, the number of extrema and the number of zero crossings must either be equal or differ at most by one; (2) At any point, the mean value of the envelope defined by the local maxima and the envelope defined by the local minima is zero. With the definition, any signal $\mathrm{x}(\mathrm{t})$ can be decomposed as follows:

First, find all the local extremum of the signal and use cubic spline to fit it as the local mean $\mathrm{m}(\mathrm{t})$ and extract the detail $\mathrm{h}(\mathrm{t})=\mathrm{x}(\mathrm{t})-\mathrm{m}(\mathrm{t})$. Regard $\mathrm{h}(\mathrm{t})$ as new $\mathrm{x}(\mathrm{t})$ and repeat the operation above until $h(t)$ satisfies the IMF conditions, then obtain the first IMF $c_{1}(t)=h(t)$. Let the residual $r_{1}(t)=x(t)-$ $h(t)$ be a new signal, repeat the above processes, obtain the other orders IMFs. The decomposition process can be stopped when $r_{n}(t)$ becomes a monotonic function from which no more IMF can be extracted. We finally obtain

$$
x(t)=\sum_{i=1}^{n} c_{i}(t)+r_{n}(t)
$$

Thus, we can achieve a decomposition of the signal into IMFs $c_{1}(t), c_{2}(t), \ldots, c_{n}(t)$, and a residue $r_{n}(t)$, which is the mean trend of $x(t)$. The IMFs include different frequency bands ranging from high to low. The frequency components contained in each frequency band are different and change with the variation of signal $x(t)$.

For each $\operatorname{IMF} c_{i}(t)$ in Eq. (1), we can always have its Hilbert transform as

$$
H\left[c_{i}(t)\right]=\frac{1}{\pi} \int_{-\infty}^{+\infty} \frac{x(\tau)}{t-\tau} d \tau
$$

With this definition, we can have an analytic signal as

$$
x_{i}(t)=c_{i}(t)+j H_{i}(\mathrm{t})=\mathrm{a}_{\mathrm{i}}(\mathrm{t}) \mathrm{e}^{j \varphi_{\mathrm{i}}(\mathrm{t})}
$$

where

$$
\begin{aligned}
a_{i}(t) & =\sqrt{c_{i}{ }^{2}(t)+H^{2}\left[c_{i}(t)\right]} \\
\varphi_{i}(t) & =\arctan \frac{H\left[c_{i}(t)\right]}{c_{i}(t)}
\end{aligned}
$$

From Eq. (5), we can have the instantaneous frequency as

$$
\omega_{i}(t)=\frac{\varphi_{i}(t)}{2 \pi}
$$

After performing the Hilbert transform to each IMF component, the original signal can be expressed as the real part $(R P)$ in the following form

$$
x(t)=R P \sum_{i=1}^{n} \mathrm{a}_{\mathrm{i}}(\mathrm{t}) \mathrm{e}^{j \varphi_{\mathrm{i}}(\mathrm{t})}
$$




\section{Independent Component Analysis}

Independent component analysis(ICA) is very closely related to the method called blind source separation(BSS). The main purpose of ICA is to find a linear representation of non-Gaussian so that the components are statistically independent. Consider $\mathrm{n}$ sources $s_{1}, s_{2}, \ldots, s_{n}$, which are statistically independent, $\mathrm{m}$ measurements from sensors $x_{1}, x_{2}, \ldots, x_{m}$, which are represented as a linear combination of sources $s_{i}$ as follows

$$
x=A s
$$

where $A$ and $s$ are unknown and $x$ is known. $A$ is the mixing matrix; The source vector $s=\left[s_{1}, s_{2}, \ldots, s_{n}\right]^{T}$; The observed vector $x=\left[x_{1}, x_{2}, \ldots, x_{m}\right]^{T}$.

Our aim is to seek a demixing matrix which recovers the source vector s from the observed vector $x$. The elements of $y$ are estimates of the observed vector $x$ which can be used to represent the observed vector $x$ as follows

$$
y=W x=W A s
$$

where $\mathrm{W}$ is the demixing matrix.

ICA is a two-step process. First step is to choose a principle, based on which a cost function is obtained. Next, a suitable method for optimizing the cost function needs to be adopted. One of the best methods is the FastICA algorithm, which uses the negentropy as the cost function to estimate $s_{i}$. Entropy is a measure of the average uncertainty in a random variable. A Gaussian variable has the maximum entropy among all random variables with equal variance. For random vector $y$, the negentropy is defined as [8]:

$$
J(y)=H\left(y_{\text {gauss }}\right)-H(y)
$$

Where $y_{\text {gauss }}$ is a Gaussian random vector with the same covariance as y. Hence, negentropy of a Gaussian random vector is the difference of entropy with the corresponding Gaussian random vector. To estimate negentropy efficiently, a simpler approximation of negentropy as follows is used:

$$
J(y) \approx c\left\{E[G(y)]-E\left[G\left(y_{\text {gauss }}\right)\right]\right\}^{2}
$$

Where $y$ is assumed to be of zero mean and unit variance; $G$ is any non-quadratic function; $c$ is a constant.

Using a fixed-point iteration scheme to find directions in which the negentropy is maximized, the demixing matrix $\mathrm{W}$ can be achieved [8].

\section{ICA-EMD Method}

The ICA- EMD method follows four operations:

1) Decompose the measured signal $X$ by using EMD, and obtain the first component IMF1.

2) Let IMF1 and $X$ be the input to the FastICA algorithm, after performing ICA, we can obtain two independent components ICA1、ICA2.

3) As the high frequency component was decomposed firstly, the higher frequency ICA among the two ICAs can be regarded as IMF1, let the other ICA be a new measured signal X1.

4) Repeat 1)、2) and 3), obtain the other orders IMFs.

\section{Experimental evaluation}

In order to verify the validity of the proposed method, the rub-impact fault occurs only in one position was conducted on a rotor test rig. The radial displacement vibration signal with rub-impact fault picked up by the displacement sensor is shown in Fig. 1(a). The rotating frequency is $47 \mathrm{~Hz}$ and the sample frequency is $2560 \mathrm{~Hz}$. The FFT spectrum of the rub-impact vibration signal is shown in Fig. 1(b). It can be demonstrated from Fig. 1(b) that the dominant frequency components of the 
rub-impact signal are the rotating frequency $47 \mathrm{~Hz}$ and its $2 \times$. However, the higher frequency components with rub-impact information are submerged in the stronger background signal.

The rub-impact vibration signal is decomposed by EMD and the result is shown in Fig. 2(a). Because of weak feature and interference of noise, it can be seen from Fig.2(a) that EMD generates the redundant components. It is very difficult to separate the rub-impact signal from the vibration signal by using EMD.

To extract the rub-impact signal, firstly the vibration signal is denoised by using lifting wavelet, then is decomposed by using ICA- EMD and the result is shown in Fig. 2(b). It can be seen from Fig. 2(b) that the component $\mathrm{C} 1$ has the obvious AM characteristic. Hence, the component C1 including the rub-impact information is just the rub-impact signal. The Hilbert envelope analysis is then applied to the component $\mathrm{C} 1$. The Hilbert envelope spectrum of the component $\mathrm{C} 1$ is shown in Fig. 3. It can be seen from Fig. 3 that there is the obvious spectrum line in the rotating frequency $47 \mathrm{~Hz}$. Therefore, the fault feature of the rub-impact vibration signal can be obtained. The other components C2 and C3 are the background signal related to the rotating frequency.

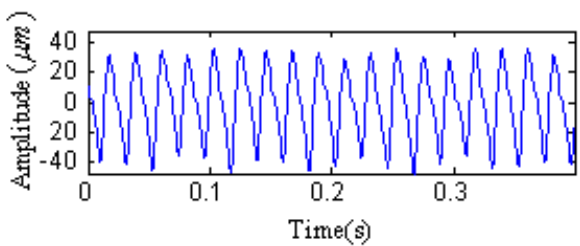

(a) Time domain waveform

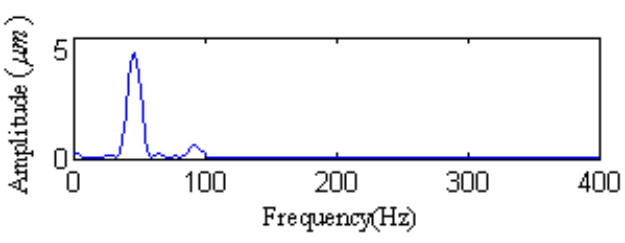

(b) Frequency spectrum

Fig. 1. The rub-impact vibration signal and its spectrum

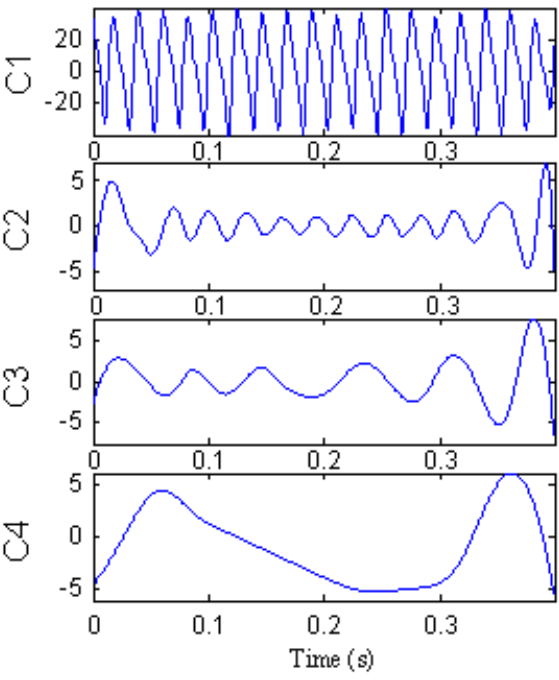

(a) Results by using EMD
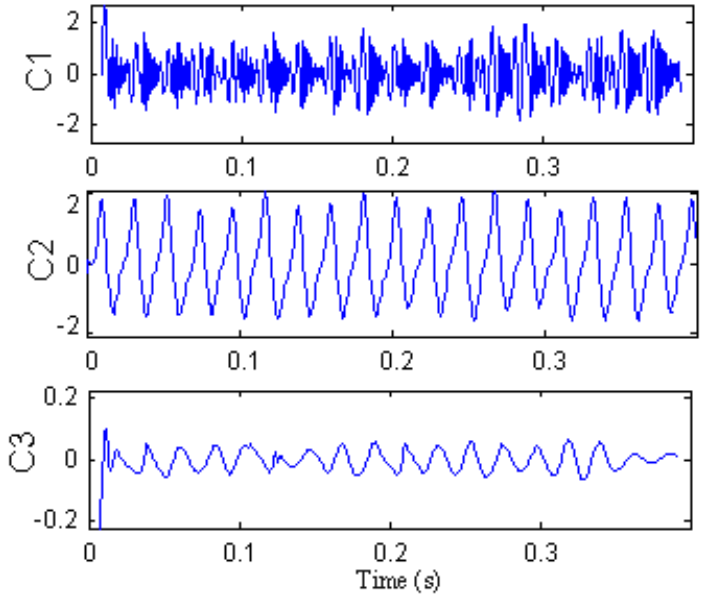

(b) Results by using the ICA-EMD

Fig. 2. Decomposition results of the rub-impact signal

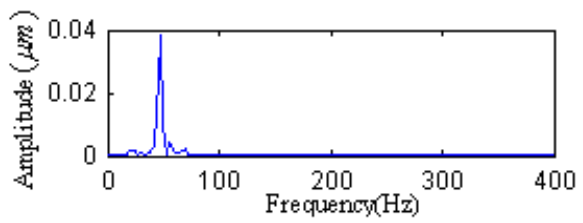

Fig. 3. Hilbert envelope spectrum of C1

In this experiment, we utilize EMD and the ICA-EMD to decompose the rub-impact signal, respectively. Fig. 2(a) and (b) are the decomposition results. We consider the first IMF which represents the main features of the vibration signal. From the results, we can find that the decomposition performance obtained by using the ICA-EMD is much better than that of the straightforward EMD. And the ICA-EMD has less redundancy over the straightforward EMD. The 
rub-impact information in the high frequency band can be extracted from the strong background signal effectively by using the ICA-EMD. Therefore, the fault feature of the vibration signal can be obtained.

\section{Conclusion}

EMD as a data driven alternative approach to the analysis of non-stationnary signals appears some drawbacks. Because the rub-impact signal is weak, it is very difficult to separate the rub-impact signal from the vibration signal including noise and background signal by using the straightforward EMD. Here we proposed solutions for the problem of component redundancy in order to obtain an algorithm with better performances. The proposed method can remove the components redundancy in EMD and improve the quality of decomposition. Compare to the straightforward EMD, the ICA-EMD can obtain a much better decomposition performance, and the AM information of the rub-impact signal can be extracted from the measured vibration signal. Experimental analysis results show that the proposed method can be applied to the feature extraction of the rotor systems with local rub-impact fault efficiently.

\section{Acknowledgements}

In this paper, the research was sponsored by the Science Research Project of Liaoning Provincial Department of Education(L2015069), the Fundamental Research Funds for the Central Universities (3132016338), the Opening Project of Technology Development Center for Polymer Processing Engineering of Guangdong Province, Guangdong Industry Technical College (201503) and the National Science Foundation(11272093).

\section{References}

[1] X.F. Fan, M.J. Zuo, Machine fault feature extraction based on intrinsic mode functions [J]. Meas. Sci. Technol, 200819 (10) 1-12.

[2] R. Li, D. He, Rotational machine health monitoring and fault detection using EMD-based acoustic emission feature quantification [J]. IEEE Trans. Instrum. Meas, 201261 (4) 990-1001.

[3] Z.J. Shen, X.F. Chen, X.L. Zhang, et al., A novel intelligent gear fault diagnosis model based on EMD and multi-class TSVM [J]. Measurement, 201245 (1) 30-40.

[4] N. E. Huang, Z Shen, S R Long, et al., The empirical mode decomposition and the Hilbert spectrum for nonlinear and nonstationary time series analysis [C]. Proceedings of the Royal Society of London,1998 454 903-995.

[5] R. Ricci, P. Pennacchi, Diagnostics of gear faults based on EMD and automatic selection of intrinsic mode functions [J]. Mech. Syst. Signal Process, 201125 (3) 821-838.

[6] C. Li, X.L. Wang, Z.Y. Tao, et al., Extraction of time varying information from noisy signals: an approach based on the empirical mode decomposition [J]. Mech. Syst. Signal Process, 2011 25(3) 812-820.

[7] R.T. Rato, M.D. Ortigueira, A.G. Batista. On the HHT, its problems, and some solutions [J]. Mechanical Systems and Signal Processing, 2008 22(6)1374-1394.

[8] A.Hyvarinen, E.Oja, Independent component analysis: algorithms and applications [J]. Neural Net, 2000 13(5)411-430.

[9] Y Yang, S Nagarajaiah, time-frequency blind source separation using independent component analysis for output-only modal identification of highly damped structures[J]. Journal of Structural Engineering, 2013 139(10)1780-1793. 
[10] Yongchao Yang, SatishNagarajaiah, Blind identification of damage in time-varying systems using independent component analysis with wavelet transform [J]. Mechanical Systems and Signal Processing, 201447 (1) 3-20. 